\title{
PRIMORDIAL BLACK HOLE PAIR CREATION PROBABILITY IN MODIFIED GRAVITATIONAL THEORY
}

\author{
B. C. Paul*and Dilip Paul \\ Department of Physics, North Bengal University, \\ Siliguri, Dist. Darjeeling, Pin : 734 013, India
}

\begin{abstract}
The probability for quantum creation of an inflationary universe with a pair of black holes is computed in a modified gravitational theory. Considering a gravitational action which includes a cosmological constant $(\Lambda)$ in addition to $\alpha R^{2}$ and $\delta R^{-1}$ terms, the probabilities have been evaluated for two different kinds of spatial sections, one accommodating a pair of black holes and the other without black hole. We adopt a technique prescribed by Bousso and Hawking to calculate the above creation probability in a semiclassical approximation with Hartle-Hawking boundary condition. Depending on the parameters in the action some new and physically interesting instanton solutions are presented here which may play an important role in the creation of the early universe. We note that the probability of creation of a universe with a pair of black holes is strongly suppressed with a positive cosmological constant when $\delta=\frac{4 \Lambda^{2}}{3}$ for $\alpha>0$ but it is more probable for $\alpha<-\frac{1}{6 \Lambda}$. It is also found that instanton solutions are allowed without a cosmological constant in the theory provided $\delta<0$.
\end{abstract}

PACS No(s). : 04.70.Dy, 98.80.Bp, 98.80.Cq.

Key Words : Gravitational instanton, primordial black hole, quantum cosmology.

\section{INTRODUCTION :}

*e-mail : bcpaul@iucaa.ernet.in 
It is now understood from the information gathered from high redshift surveys of supernovae to Wilkinson Microwave Anisotropy Probe ( WMAP ) data that the present universe is accelerating [1]. The universe might have also emerged from an inflationary era in the past predicted from COBE. In the last two decades a number of literature appeared which explored early universe with an inflationary phase [2]. Inflation not only opens up new avenues in the interface of particle physics and cosmology but also solves some of the outstanding problems not understood in the standard bigbang cosmology. Most successful theory developed so far is based on the dynamics of scalar field with a suitable potential which drives inflation. In order to explain present acceleration in the universe, various cosmological models have been proposed with exotic fields that appeared with a new gravitational physics considering theories other than scalar field, e.g., chaplygin gas [3], phantom fields [4] etc. It may be pointed out here that an alternative theory with a modification to the Einstein-Hilbert action has been employed to obtain early inflation instead of a scalar field. It is known that a modification to the Einstein-Hilbert action with higher order terms in curvature invariants that are become effective in the high curvature region lead to modifications of the standard cosmology admitting a de Sitter universe at early times [5]. In the same way it is important to look for modifications of the Einstein gravitational action with terms that might be important at extremely low curvature region to explain the present cosmic acceleration. Recently, various cosmological models are proposed to explain the cosmic speed up with a modification of the Einstein Hilbert action. Carroll et al. [6] considered a gravitational action of the form

$$
I=\frac{1}{2 \kappa^{2}} \int d^{4} x \sqrt{-g}\left[R-\frac{\mu^{4}}{R}\right]
$$

where $R$ is the Ricci Scalar, $\kappa^{2}=8 \pi G$, and $\mu$ is a mass scale of the order of Hubble scale, to construct such models of the universe. In the literature [7] a number of cosmological models 
are appeared with the above action to construct an alternative theory of dark energy and dark matter. However, in the theory de Sitter space is always unstable. It has been shown [8] that adding a $\alpha R^{2}$ term to the action allows a stable de Sitter space if the size of the baby universe is bigger than a size determined by $\mu^{2}$ in the action. Further, the theory containing both the $1 / R$ and $R^{2}$ terms has better chance of passing solar system tests than the theory based on simple $1 / R$ corrections [9]. It is also important to explore the cosmological issues in the framework of new theories which are important at the present epoch. The astrophysical objects, for example black holes need to be investigated in these theoretical framework. The mass of these objects may be greater than the solar mass or even less. It is known in stellar physics that a blackhole is the ultimate corpse of a collapsing star when its mass exceeds twice the mass of the Sun. Another kind of black holes are important in cosmology which might have formed due to quantum fluctuations of matter distribution in the early universe. These are termed as topological blackholes having mass many times smaller than the the solar mass. In particular, black holes whose mass $\left(\sim 10^{15} \mathrm{~g}\right)$ have life times comparable to the age of the universe so that they would be evaporating at present which is Hawking radiation [10]. It may provide sources of ultra high energy cosmic rays including extragalactic gamma-rays [11] and other astrophysical phenomena whose mechanisms have not been solved completely. In addition to that, the relic black hole might contribute to the energy density of the present universe and solve the dark matter problem [12]. Bousso and Hawking [13] (in short, BH) calculated the probability of the quantum creation of a universe with a pair of primordial black holes (in short, $\mathrm{PBH})$ in $(3+$ 1) dimensional universe. To compute the probablity measure, $\mathrm{BH}$ considered two different Euclidean space-time : (i) a universe with space-like sections with $S^{3}$ - topology and (ii) a universe with space-like section with $S^{1} \times S^{2}$ - topology, as is obtained in the Schwarzschildde Sitter solution. The first kind of spatial structure describes an inflationary (de Sitter) 
universe without black hole while the second kind describes a Nariai universe [14], an inflationary universe with a pair of black holes. Considering a massive scalar field which provided an effective cosmological constant for a while through a slow-rolling potential (mass-term) BH studied the problem. Chao [15] studied the creation of a primordial black hole and Green and Malik [16] studied the primordial black holes production during reheating. Paul et al. [17] following the approach of $\mathrm{BH}$ studied the probability of creation of $\mathrm{PBH}$ including $R^{2}$-term in the Einstein action and found that the probability is very much suppressed in the $R^{2}$-theory. Paul and Saha [18] studied probability of creation of a pair of black hole with higher order Lagrangian i.e., considering higher loop contributions into the effective action that are higher than quadratic in $R$. Recently, the probability for quantum creation of an inflationary universe with a pair of black hole in a gravitational theory which includes a cosmological constant $(\Lambda)$ in addition to $\left(\delta R^{-1}\right)$ term is evaluated [19]. In this paper we investigate the probability for primordial black hole pair creation in a modified gravity by adding an extra term $\alpha R^{2}$ to the action given by (1) in the presence of a cosmological constant. The above theory is important as it may be regarded as the synthesis of the theories one needs for early and late inflation, although it remains to be understood how the universe transits from early inflating phase to the present accelerating phase.

Consequently it is also important to study the effects of these terms to study quantum creation of a universe with a pair of $\mathrm{PBH}$. We calculate the probabilities for the creation of a universe with two types of topology namely, $R \times S^{3}$ - topology and $R \times S^{1} \times S^{2}$ topology, where the later accommodates a pair of primordial black holes. To calculate the probabilities for these spatial topologies, we use a semiclassical approximation for the evaluation of the Euclidean path integrals. The condition that a classical spacetime should emerge, to a good approximation, at a large Lorentzian time was selected by a choice of 
the path of the time parameter $\tau$ along the $\tau^{R e}$ axis from 0 to $\frac{\pi}{2 H}$ and then continues along the $\tau^{i m}$ axis. The wave-function of the universe in the semiclassical approximation is given by

$$
\Psi_{o}\left[h_{i j}, \Phi_{\partial M}\right] \approx \sum_{n} A_{n} e^{-I_{n}}
$$

where the sum is over the saddle points of the path integral, and $I_{n}$ denotes the corresponding Euclidean action. The probability measure of the creation of $\mathrm{PBH}$ is

$$
P\left[h_{a b}, \Phi_{\partial M}\right] \sim e^{-2 I^{R e}}
$$

where $h_{a b}$ is the boundary metric and $I^{R e}$ is the real part of the action corresponding to the dominant saddle point, i.e. the classical solution satisfying the Hartle-Hawking (henceforth, HH) boundary conditions [20]. It was believed that all inflationary models lead a density parameter to $\Omega_{o} \sim 1$ to a great accuracy. This view has been modified after it was discovered that there is a special class of inflaton effective potentials which may lead to a nearly homogeneous open universe with $\Omega_{o} \leq 1$ at the present epoch. Cornish et al. $[21,22]$ studied the problem of pre-inflationary homogeneity and outlined the possibility of creation of a small, compact, negatively curved universe. We show that a universe with $S^{3}$-topology may give birth to an open inflation similar to that obtained in Ref. 23.

The paper is organised as follows : in sec. II, the gravitational action for a higher derivative gravity is written and gravitational instanton solutions are presented and in sec. III, the action is used to estimate the relative probability for the two types of the universes one with $\mathrm{PBH}$ and the other without PBH. Finally, in sec. IV we give a brief discussion.

II. GRAVITATIONAL ACTION AND INSTANTON SOLUTIONS WITH OR WITHOUT A PAIR OF PRIMORDIAL BLACK HOLE :

We consider Euclidean action which is given by

$$
I_{E}=-\frac{1}{16 \pi} \int d^{4} x \sqrt{g} f(R)-\frac{1}{8 \pi} \int_{\partial M} d^{3} x \sqrt{h} K f^{\prime}(R)
$$


where $g$ represents the 4-dimensional Euclidean metric, $\alpha$ and $\delta$ are dimensional parameters, $\Lambda$ represents the 4-dimensional cosmological constant and $f(R)$ is a polynimial function in Ricci Scalar $R$. The second term in the action is the gravitational surface term where $h_{i j}$ is the boundary metric and $K=h^{i j} K_{i j}$ is the trace of $K_{i j}$, the second fundamental form of the boundary $\partial M$ in the given metric $g$. The second term is the contribution from $\tau=0$ back in the action. It vanishes for a universe with topology $S^{3}$, but gives a non vanishing contribution for topology $S^{1} \times S^{2}$.

\section{(i) $S^{3}$-Topology, the de Sitter spacetime :}

In this section, we first derive the field equations from the Eucliden action and then explore instanton solutions in the modified gravitational theories. We now look for a solution with spacelike section having $S^{3}$-topology. The corresponding four dimensional metric ansatz in this case is given by

$$
d s^{2}=d \tau^{2}+a^{2}(\tau)\left[d x_{1}^{2}+\sin ^{2} x_{1} d \Omega_{2}^{2}\right]
$$

where $a(\tau)$ is the scale factor of a four dimensional universe and $d \Omega_{2}^{2}$ is a line element on the unit two sphere. The scalar curvature is given by

$$
R=-6\left[\frac{\ddot{a}}{a}+\left(\frac{\dot{a}^{2}}{a^{2}}-\frac{1}{a^{2}}\right)\right] .
$$

where an overdot denotes differentiation with respect to $\tau$. We rewrite the action (3), including the constraint through a Lagrangian multiplier $\beta$ and obtain

$$
I_{E}=-\frac{\pi}{8} \int\left[f(R) a^{3}-\beta\left(R+6 \frac{\ddot{a}}{a}+6 \frac{\dot{a}^{2}-1}{a^{2}}\right)\right] d \tau-\frac{1}{8 \pi} \int_{\partial M} d^{3} x \sqrt{h} K f^{\prime}(R) .
$$

Varying the action with respect to $\mathrm{R}$, we determine

$$
\beta=a^{3} f^{\prime}(R)
$$

Substituting the above equation, treating $a$ and $R$ as independent variables we get

$$
I_{E}=-\frac{\pi}{8} \int_{\tau=0}^{\tau \frac{\pi}{2 H}}\left[a^{3} f(R)-f^{\prime}(R)\left(a^{3} R-6 a \dot{a}^{2}-6 a\right)+6 a^{2} \dot{a} \dot{R} f^{\prime \prime}(R)\right] d \tau
$$




$$
-\frac{3 \pi}{4}\left[\dot{a} a^{2} f^{\prime}(R)\right]_{\tau=0}
$$

here we have eliminated $\ddot{a}$ term in the action by integration by parts. The field equations are now obtained by varying the action with respect to $a$ and $R$ respectively, giving

$$
\begin{gathered}
f^{\prime \prime}(R)\left[R+6 \frac{\ddot{a}}{a}+6 \frac{\dot{a}^{2}-1}{a^{2}}\right]=0, \\
2 f^{\prime \prime \prime}(R) \dot{R}^{2}+2 f^{\prime \prime}(R)\left[\ddot{R}+2 \frac{\dot{a}}{a} \dot{R}\right]+f^{\prime}(R)\left[4 \frac{\ddot{a}}{a}+2 \frac{\dot{a}^{2}}{a^{2}}-\frac{2}{a^{2}}+R\right]-f(R)=0 .
\end{gathered}
$$

We now consider $f(R)=R-2 \Lambda+\alpha R^{2}+\frac{\delta}{R}$ to solve Eqs. (8)-(9). An instanton solution is obtained which is given by

$$
a=\frac{1}{H} \sin H \tau
$$

where $R=12 H^{2}$ and $H$ can be determined in terms of the parameters $\delta, \Lambda$ from the constraint equation which emerged from eq.(9) :

$$
48 H^{4}-16 \Lambda H^{2}+\delta=0
$$

We note following from the Eq. (11) : (i) A real Hubble parameter is permitted for $\delta \leq \frac{4 \Lambda^{2}}{3}$, (ii) two classes of instantons are allowed for $0<\delta<\frac{4 \Lambda^{2}}{3}$, (iii) Instanton solution with $H=\sqrt{\frac{\Lambda}{6}}$ is obtained when $\delta=\frac{4 \Lambda^{2}}{3}$, (iv) Instanton solution with $H=\sqrt{\frac{\Lambda}{3}}$ is obtained when $\delta=0$, and (v) Instanton solution is permitted even with $\Lambda=0$ when $\delta<0$.

It is evident that the instanton solution (10) obtained here satisfies the HH no boundary conditions viz., $a(0)=0, \dot{a}(0)=1$. One can choose a path along the $\tau^{R e}$ axis to $\tau=\frac{\pi}{2 H}$, the solution describes half of the Euclidean de Sitter instanton $S^{3}$. Analytic continuation of the metric (4) to Lorentzian region, $x_{1} \rightarrow \frac{\pi}{2}+i \sigma$, gives

$$
d s^{2}=d \tau^{2}+a^{2}(\tau)\left[-d \sigma^{2}+\cosh ^{2} \sigma d \Omega_{2}^{2}\right]
$$

which is a spatially inhomogeneous de Sitter like metric. However, if one sets $\tau=i t$ and 
$\sigma=i \frac{\pi}{2}+\chi$, the metric becomes

$$
d s^{2}=-d t^{2}+b^{2}(t)\left[d \chi^{2}+\sinh ^{2} \chi d \Omega_{2}^{2}\right]
$$

where $b(t)=-i a(i t)$. The line element (12) now describes an open inflationary universe. The real part of the Euclidean action corresponding to the solution calculated by following the complex contour of $\tau$ suggested by $\mathrm{BH}$ is given by

$$
I_{S^{3}}^{R e}=-\frac{\pi}{8}\left[\frac{1728 \alpha H^{6}+144 H^{4}-24 \Lambda H^{2}+\delta}{18 H^{6}}\right]
$$

With the chosen path for $\tau$, the solution describes half the de Sitter instanton with $S^{4}$ topology, joined to a real Lorentzian hyperboloid of topology $R^{1} \times S^{3}$. It can be joined to any boundary satisfying the condition $a_{\partial M}>0$. For $a_{\partial M}>H^{-1}$, the wave function oscillates and predicts a classical space-time. We note the following cases :

(a) $S^{3}$ solution obtained by $\mathrm{BH}[13]$ is recovered when $\delta=0, \alpha=0$.

(b) $\delta=0, \alpha \neq 0$, corresponds to the instanton solution obtained by Paul et al. [17].

(c) $\delta \neq 0, \alpha=0, \Lambda \neq 0$, corresponds to the solution obtained by us recently [19] and the action reduces to $I=-\frac{8 \pi}{\sqrt{-3 \delta}}$ for a vanishing cosmological constant. It is evident here that a realistic solution is obtained with a negative $\delta$ only.

(d) $\delta \neq 0, \alpha \neq 0$ and $\Lambda=0$, one obtains action which is $I=-4 \pi\left[3 \alpha+\frac{2}{\sqrt{-3 \delta}}\right]$. But the above solution also demands $\delta<0$.

(e) $\delta=\frac{4 \Lambda^{2}}{3}$, we get the action which becomes $I=-12 \pi \alpha-\frac{2 \pi}{\Lambda}$. It is interesting if $\alpha<0$.

(ii) Spacelike sections $S^{1} \times S^{2}$, the Nariai spacetime:

We now look for a solution with spacelike sections $S^{1} \times S^{2}$ as this topology accommodates a pair of black holes. The corresponding ansatz for $(1+1+2)$ dimensions is given by

$$
d s^{2}=d \tau^{2}+a^{2}(\tau) d x^{2}+b^{2}(\tau) d \Omega_{2}^{2}
$$


where $a(\tau)$ is the scale factor of $S^{1}$-surface and $b(\tau)$ is the scale factor of the two sphere. The metric for the two-sphere is given by the metric

$$
d \Omega_{2}^{2}=d \theta^{2}+\sin ^{2} \theta d \phi^{2}
$$

The scalar curvature is given by

$$
R=-\left[2 \frac{\ddot{a}}{a}+4 \frac{\ddot{b}}{b}+2\left(\frac{\dot{b}^{2}}{b^{2}}-\frac{1}{b^{2}}\right)+4 \frac{\dot{a} \dot{b}}{a b}\right] .
$$

The Euclidean action (3) becomes

$$
\begin{gathered}
I_{E}=-\frac{\pi}{2} \int\left[f(R) a b^{2}-\beta\left(R+2 \frac{\ddot{a}}{a}+4 \frac{\ddot{b}}{b}+4 \frac{\dot{a} \dot{b}}{a b}+2 \frac{\dot{b}^{2}}{b^{2}}-\frac{2}{b^{2}}\right)\right] d \tau \\
-\frac{1}{8 \pi} \int_{\partial M} \sqrt{h} d^{3} x K f^{\prime}(R) .
\end{gathered}
$$

One can determine $\beta$ as is done before and obtains

$$
\begin{gathered}
I_{S^{1} \times S^{2}}=-\frac{\pi}{2} \int_{\tau=0}^{\tau_{\partial M}}\left[f(R)-f^{\prime}(R)\left(R-4 \frac{\dot{a} \dot{b}}{a b}-2 \frac{\dot{b}^{2}}{b^{2}}-\frac{2}{b^{2}}\right)+2 f^{\prime \prime}(R) \dot{R}\left(\frac{\dot{a}}{a}+2 \frac{\dot{b}}{b}\right)\right] \\
a b^{2} d \tau-\pi\left[\left(\dot{a} b^{2}+2 a b \dot{b}\right) f^{\prime}(R)\right]_{\tau=0} .
\end{gathered}
$$

Variation of the action with respect to $R, a$ and $b$ respectively are given by the following equations

$$
\begin{gathered}
f^{\prime \prime}(R)\left[R+2 \frac{\ddot{a}}{a}+4 \frac{\ddot{b}}{b}+4 \frac{\dot{a} \dot{b}}{a b}+2 \frac{\dot{b}^{2}}{b^{2}}-\frac{2}{b^{2}}\right]=0 \\
2 f^{\prime \prime \prime}(R) \dot{R}^{2}+2 f^{\prime \prime}(R)\left[\ddot{R}+2 \dot{R} \frac{\dot{b}}{b}\right]+f^{\prime}(R)\left[R+4 \frac{\ddot{b}}{b}+2 \frac{\dot{b}^{2}-1}{b^{2}}\right]-f(R)=0, \\
2 f^{\prime \prime \prime}(R) \dot{R}^{2}+2 f^{\prime \prime}(R)\left[\dot{R}\left(\frac{\dot{a}}{a}+\frac{\dot{b}}{b}\right)+\ddot{R}\right]+f^{\prime}(R)\left[R+2 \frac{\ddot{a}}{a}+2 \frac{\ddot{b}}{b}+2 \frac{\dot{a} \dot{b}}{a b}\right] \\
-f(R)=0 .
\end{gathered}
$$

Let us now consider $f(R)=R-2 \Lambda+\alpha R^{2}+\frac{\delta}{R}$. Eqs. (18)-(20) admit an instanton solution which is given by

$$
a=\frac{1}{H_{o}} \sin \left(H_{o} \tau\right), \quad b=H_{o}^{-1}
$$




$$
R=4 H_{o}^{2}
$$

where $H_{o}$ satisfies the constraint equation

$$
16 H_{o}^{4}-16 \Lambda H_{o}^{2}+3 \delta=0
$$

We note the following : (i) when $0<\delta<\frac{4 \Lambda^{2}}{3}$, two classes of instanton solutions are permitted, (ii) $\delta=0$, instanton solution with $H_{o}^{2}=\Lambda$ is obtained, (iii) $\delta=\frac{4}{3} \Lambda^{2}$ leads to an instanton solution with $H_{o}^{2}=\frac{\Lambda}{2}$, (iv) $\Lambda=0$, permits instanton with $H_{o}^{2}=\sqrt{-\frac{3 \delta}{16}}$ when $\delta<0$.

The above instanton solution (21) satisfies the HH boundary conditions $a(0)=0, \dot{a}(0)=$ $1, b(0)=b_{o}, \dot{b}(0)=0$. Analytic continuation of the metric (14) to Lorentzian region, i.e., $\tau \rightarrow i t$ and $x \rightarrow \frac{\pi}{2}+i \sigma$ yields

$$
d s^{2}=-d t^{2}+c^{2}(t) d \sigma^{2}+H_{o}^{-2} d \Omega_{2}^{2}
$$

where $c(t)=-i a(i t)$. In this case the analytic continuation of time and space do not give an open inflationary universe. The corresponding Lorentzian solution is given by

$$
\begin{gathered}
\left.a\left(\tau^{I m}\right)\right|_{\tau^{R e}=\frac{\pi}{2 H_{o}}}=H_{o}^{-1} \cosh H_{o} \tau^{I m}, \\
\left.b\left(\tau^{I m}\right)\right|_{\tau^{R e}}=\frac{\pi}{2 H_{o}}=H_{o}^{-1}
\end{gathered}
$$

Its space like sections can be visualised as three spheres of radius $H_{o}^{-1}$ with a hole of radius $b=H_{o}^{-1}$ punched through the north and south poles. The physical interpretation of the solution is that of two - spheres containing two black holes at opposite ends. The black holes have the radius $H_{o}^{-1}$ which accelerates away from each other with the expansion of the universe. This describes half of a Lorentzian Nariai universe. The real part of the action can be determined now following the contour suggested by $\mathrm{BH}$ [13], and it is given by

$$
I_{S^{1} \times S^{2}}^{R e}=-\frac{\pi}{8 H_{o}^{2}}\left[64 \alpha H_{o}^{6}+16 H_{o}^{4}-8 \Lambda H_{0}^{2}+\delta\right]
$$


The solution given by eq.(24) describes a universe with two black holes at the poles of a two sphere. It may be pointed out here that the contribution of the integrand in the action (17) for the instanton vanishes and the non-zero contribution of the action here arises just from the boundary term only. We note the following :

(a) $\delta=0, \alpha=0$, corresponds to the action obtained by $\mathrm{BH}[13]$.

(b) $\delta=0, \alpha \neq 0$ corresponds to $I=-8 \pi \alpha-\frac{\pi}{\Lambda}$, it reduces to a case discussed by Paul et al. [17].

(c) $\delta \neq 0, \alpha=0, \Lambda=0$, the action is $I=-\frac{16 \pi}{3 \sqrt{-3 \delta}}$, it is real for $\delta<0$, which corresponds to the result obtained by us [19].

(d) $\delta \neq 0, \alpha \neq 0$ and $\Lambda=0$ corresponds to an action which is

$$
I_{S^{1} \times S^{2}}^{R e}=-\frac{8 \pi}{3}\left(3 \alpha+\frac{2}{\sqrt{-3 \delta}}\right)=\frac{2}{3} I_{S^{3}}^{R e}
$$

In this case a physically realistic solution is obtained for $\delta<0$.

(e) $\delta=\frac{4 \Lambda^{2}}{3}$, the action becomes

$$
I_{S^{1} \times S^{2}}^{R e}=-\frac{4 \pi}{3}\left(6 \alpha+\frac{1}{\Lambda}\right)=\frac{2}{3} I_{S^{3}}^{R e}
$$

\section{THE PROBABILITY FOR THE PRIMORDIAL BLACK HOLES PAIR CREATION :}

In the previous section we have calculated the actions for inflationary universe with or without a pair of black holes. We now compare the probability measure in the two cases. The probability for creation of a de Sitter universe is determined from the action (7). The probability for nucleation of an inflationary universe without a pair of Black holes is given by

$$
P_{S^{3}} \sim e^{\frac{\pi}{9}\left[\frac{216 \alpha H^{4}+12 H^{2}-\Lambda}{H^{4}}\right]}
$$

However for an inflationary universe with a pair of black holes the corresponding probability 
of nucleation can be obtained from the action (17). The corresponding probability is

$$
P_{S^{1} \times S^{2}} \sim e^{\frac{2 \pi}{3}\left[\frac{24 \alpha H_{o}^{4}+4 H_{0}^{2}-\Lambda}{H_{0}^{4}}\right]} .
$$

For simplicity to compare the probabilities we consider spacial cases :

(a) The probabilities obtained by Bousso and Hawking [13] is recovered when both $\delta$ and $\alpha$ are set zero. The probabilities are

$$
P_{S^{3}} \sim e^{\frac{3 \pi}{\Lambda}}, \quad P_{S^{1} \times S^{2}} \sim e^{\frac{2 \pi}{\Lambda}} .
$$

In this case the probability for a universe with $\mathrm{PBH}$ is less than that without $\mathrm{PBH}$. The solution cannot admit a negative cosmological constant.

(b) The probabilities evaluated by Paul et al. [17] are recovered here with $\delta=0$. The probabilities are

$$
P_{S^{3}} \sim e^{\frac{3 \pi}{\Lambda}+24 \pi \alpha}, \quad P_{S^{1} \times S^{2}} \sim e^{\frac{2 \pi}{\Lambda}+16 \pi \alpha} .
$$

In this case with a positive cosmological constant and $\alpha>0$, the de Sitter universe is more probable. However for $\alpha<-\frac{1}{8 \Lambda}$, the probability for a universe with $\mathrm{PBH}$ is more. However, the case $\alpha<0$ leads to a classical instability in $R^{2}$ theory.

(c) For $\delta \neq 0$ and $\alpha=0$, the solutions obtained in Ref. 19 are recovered. There are two branches of solutions admitting two different instanton solutions. In one case the probabilities are given by

$$
P_{S^{3}} \sim e^{\frac{6 \pi}{\left(\Lambda+\sqrt{\Lambda^{2}-\frac{3 \delta}{4}}\right)^{3}}\left[2 \Lambda\left(\Lambda+\sqrt{\Lambda^{2}-\frac{3 \delta}{4}}\right)-\delta\right]}, \quad P_{S^{1} \times S^{2}} \sim e^{\frac{4 \pi}{\left(\Lambda+\sqrt{\Lambda^{2}-\frac{3 \delta}{4}}\right)^{3}}\left[2 \Lambda\left(\Lambda+\sqrt{\Lambda^{2}-\frac{3 \delta}{4}}\right)-\delta\right]}
$$

for $\delta<\frac{4 \Lambda^{2}}{3}$, and in the other case the probabilities are

$$
P_{S^{3}} \sim e^{\frac{6 \pi}{\left(\Lambda-\sqrt{\Lambda^{2}-\frac{3 \delta}{4}}\right)^{3}}\left[2 \Lambda\left(\Lambda-\sqrt{\Lambda^{2}-\frac{3 \delta}{4}}\right)-\delta\right]}, \quad P_{S^{1} \times S^{2}} \sim e^{\frac{4 \pi}{\left(\Lambda-\sqrt{\Lambda^{2}-\frac{3 \delta}{4}}\right)^{3}}\left[2 \Lambda\left(\Lambda-\sqrt{\Lambda^{2}-\frac{3 \delta}{4}}\right)-\delta\right]}
$$


for $0<\delta<\frac{4 \Lambda^{2}}{3}$. It is evident from eqs. (30) and (31) that the creation of a universe without $\mathrm{PBH}$ is more probable than with $\mathrm{PBH}$ for $\delta<\frac{4 \Lambda^{2}}{3}$ with a positive cosmological constant. It admits negative $\delta$ also. However, the probability for creation of a universe with a pair of $\mathrm{PBH}$ is favoured in the first case when one considers a negative cosmological constant. In the second case, the probability of creation of a universe with a pair of $\mathrm{PBH}$ is favoured with a positive cosmological constant for $0<\delta<\Lambda^{2}$, but it is suppressed for $\Lambda^{2}<\delta<\frac{4 \Lambda^{2}}{3}$. In the second case no instanton solution exists with a negative cosmological constant.

(d) For $\delta=\frac{4 \Lambda^{2}}{3}$ and $\alpha \neq 0$, the probabilities are given by

$$
P_{S^{3}} \sim e^{\frac{4 \pi}{\Lambda}+24 \pi \alpha}, \quad P_{S^{1} \times S^{2}} \sim e^{\frac{8 \pi}{3 \Lambda}+16 \pi \alpha} .
$$

In this case a positive cosmological constant and $\alpha>0$ leads to a de Sitter universe creation more probable than a universe with a pair of black holes. However, for $\alpha<-\frac{1}{6 \Lambda}$, we note that creation of a universe with a $\mathrm{PBH}$ is more probable.

(e) For a theory without cosmological constant, the evaluated probabilities are

$$
P_{S^{3}} \sim e^{24 \pi \alpha+\frac{16 \pi}{\sqrt{-3 \delta}}}, \quad P_{S^{1} \times S^{2}} \sim e^{16 \pi \alpha+\frac{32 \pi}{3 \sqrt{-3 \delta}}} .
$$

It is evident that negative values for the parameter $\delta$ are permitted to obtain a physically relevant solution. For $\delta<0$, we note that a universe without a $\mathrm{PBH}$ is more probable for positive values of the prameter $\alpha$, however, it is less probable if one considers a negative $\alpha$ satisfying the inequality $\alpha<-\frac{2}{3} \frac{1}{\sqrt{-3 \delta}}$, determined by a negative $\delta$.

\section{DISCUSSIONS :}

In this letter we estimated and compared the probability for creation of a universe with a primordial black hole $(\mathrm{PBH})$ pair with a universe without such $\mathrm{PBH}$ pair considering a modified theory of gravity. In section II, we obtained gravitational instanton solutions in the two cases : (i) a universe with $R \times S^{3}$ - topology and (ii) a universe with $R \times S^{1} \times$ 
$S^{2}$ - topology respectively. The Euclidean action is then evaluated corresponding to the instanton solutions using $\mathrm{BH}$ technique [13]. We found that the probability of a universe with $R \times S^{3}$ topology turns out to be lower than a universe with topology $R \times S^{1} \times S^{2}$ in some specified restricted conditions. It may be mentioned here that one gets a regular instanton in $S^{4}$ - topology if there is no black hole. The existence of black hole restricts such a regular topology. The results obtained here on the probability of creation of a universe with a pair of primordial black holes are found to be strongly suppressed depending on the parameter $\delta$ decided by the cosmological constant in some cases which are determined here. It is noted that the probability for a universe with $\mathrm{PBH}$ is strongly suppressed for small values of $\delta$ lying in the sector $0<\delta<\frac{4 \Lambda^{2}}{3}$ in the presence of a positive cosmological constant. We note an interesting solution here in the framework of the modified gravitational action with inverse power of $R$-theory, which admits de Sitter instantons with $S^{3}$ and $S^{1} \times S^{2}$ topologies even without a cosmological constant. However, a negative $\delta$ is required if one considers a theory without a cosmological constant. In this case the action is similar to that considered by Carroll et al. [6] to obtain an accelerating universe today. We also note another case where a de Sitter universe is less probable if $\alpha<-\frac{1}{6 \Lambda}$. The result obtained earlier with curvature square term in the action in Ref. [17] given by eq. (29) now can be compared with eq. (32). It is evident that although the effect of inverse square term in the action enhances the probability, the condition to get a universe with a $\mathrm{PBH}$ remained unaltered in the theories here. It is interesting to note here that analytic continuation of a $R \times S^{3}$ metric considered here to Lorentzian region leads to an open 3 - space. One obtains Hawking-Turok [23] type open inflationary universe in this case. In the other type of topology an open inflation section of the universe is not permitted. A detail study of an open inflationary universe will be discussed elsewhere. Thus in a modified Lagrangian with inverse power in $R$-theory, quantum creation of $\mathrm{PBH}$ seems to 
be suppressed in the minisuperspace cosmology for some values of the parameters in the action, which are determined here. Another new result obtained here is that gravitational instanton solution may be obtained even with a negative cosmological constant which is not permitted in the case considered by $\mathrm{BH}[13]$.

Acknowledgement : $\mathrm{BCP}$ would like to thank Inter-University Centre for Astronomy and Astrophysics (IUCAA), Pune for awarding Visiting Associateship of IUCAA. Authors like to thank S. Mukherjee for providing facilities of the IUCAA Reference Centre and Physics Department at North Bengal University to carry out a part of the work. This work is partially supported by University Grants Commission, New Delhi.

\section{References}

[1] D. N. Spergel et al., Astrophys. J. Suppl. 148, 175 (2003); L. Verde et al., Astrophys. J. Suppl. 148, 195 (2003); S. Perlmutter et al., Astrophys. J. 517, 565 (1999); Bull. Am. Astron. Soc. 29, 1351 (1997), Astrophys. J.517, 565 (1999), Measuring cosmology with Supernovae, astro-ph/0303428.

[2] Hawking radiation A. A. Starobinsky, Phys. Lett. 99, 24 (1980); A. H. Guth, Phys. Rev. D 23, 347 (1981); A. D. Linde, Phys. Lett. B 108, 389 (1982); A. Albreht and P. J. Steinhardt, Phys. Rev. Lett. 48, 1220 (1982).

[3] N. Bilic, R. J. Lindebaum, G. B. Tupper and R. D. Viollier, Inhomogeneous Chaplygin gas cosmology, astro-ph/0310181; L.P. Chimento, Phys. Re. D 69, 123517 (2004); O. Bertolami, Challenges on the generalized chaplygin gas cosmology, astro-ph/0403310; T. Barreiro and A. A. Sen, Phys. Rev. D 70, 124013 (2004).

[4] P. Singh, M. Sami and N. Dadhich, Phys. Rev., D 68, 023522 (2003). 
[5] T. V. Ruzmaikina and A. A. Ruzmaikin, Sov. Phys. JETP 30, 372 (1970); A. A. Starobinsky, Phys. Lett., 99, 24 (1980); A. A. Starobinsky, Sov. Astron. Lett., 9, 302 (1983); R. Fabbri and M. D. Pollock, Phys. Lett. 125 B 445, (1983); J. D. Barrow and A. C. Ottewill, J. Phys. A 16, 2757 (1983); S. W. Hawking and J. C. Luttrell, Nuc. Phys., B 247, 250 (1984); A. A. Starobinsky, JETP Lett., 42, 15 (1985); L. A. Kofman, A. D. Linde and A. A. Starobinsky, Phys. Lett., 157 B, 361 (1985); A. Vilenkin, Phys. Rev. D 32, 2511 (1985); M. B. Mijic, M. S. Morris and W. Suen, Phys. Rev. D 34, 2934 (1986); S. Gottlober and V. Muller, Class. Quantum Grav. 3, 183 (1986); J. D. Barrow and S. Cotsakis, Phys. Lett. B 214, 515 (1988); B. C. Paul, D. P. Datta and S. Mukherjee, Mod. Phys. Lett. A 3, 843 (1988); J. D. Barrow and S. Cotsakis, Phys. Lett. B 258, 299 (1991); G. Magnano and L. M. Sokolowski, Phys. Rev. D 50, 5039 (1994); A. Dobado and A. L. Maroto, Phys. Rev. D 52, 1895 (1990); H. J. Schmidt, Astron. Nachr. 311, 165 (1990); S. Capozzielo, S. Carloni and A. Troisi, astro-ph/0303041.

[6] S. M. Carroll, V. Duvvuri, M. Trodden and M. S. turner, Phys. Rev. D 70, 043528 (2004), astro-ph/0306438.

[7] S. Capozzielo, V. F. Cardone and A. Troisi, Phys. Rev. D 71 , 043503 (2005); S. Das, N. Banerjee and N. Dadhich, Curvature driven acceleration : an utopia or a reality?, astro-ph/0505096; I. Brevik, S. Nojiri, S. D. Odintsov and I Vanzo, Phys. Rev. D 70, 043520 (2004); S. Nojiri and S. D. Odintsov, Phys. Lett. B 576, 5 (2003); S. Nojiri and S. D. Odintsov, Phys. Rev. D 68, 123512 (2003); M. Abdalla, S. Nojiri and S. D. Odintsov, Class. Quantum Grav. 22, L 35 (2005).

[8] V. Faraoni, Phys. Rev. D 72, 061501 (R) (2005). 
[9] S. Nojiri and S. D. Odintsov, Phys. Rev. D 68, 123512 (2003); Gen. Rel. Grav. 36, 1765 (2004).

[10] S. W. Hawking, Comm. Math. Phys. 43, 199 (1975).

[11] B. J. Carr, Astrophys. J. 201, 1 (1975); D. Page and S. Hawking, Astrophys. J. 206, 1 (1976); B. J. Carr Astrophys. J. 206, 8 (1976); J. A. MacGibbon and B. J. Carr, Astrophys. J. 371, 447 (1991).

[12] J. A. MacGibbon, Nature 329, 308 (1987).

[13] R. Bousso and S. W. Hawking, Phys. Rev. D 52, 5659 (1995).

[14] H. Nariai, Sci. Rep. Tohoku Univ. 35, 62 (1951).

[15] W. Z. Chao, Int. J. Mod. Phys. D6, 199 (1997).

[16] A. M. Green and K. A. Malik, Phys. Rev. D 64, 021301 (2001).

[17] B. C. Paul, G. P. Singh, A. Beesham and S. Mukherjee, Mod. Phys. Letts. A 13, 2289 (1998).

[18] B. C. Paul and A. Saha, Int. J. Mod. Phys. D 11, 493 (2002).

[19] D. Paul and B. C. Paul,Phys. Rev. D 72, 064012 (2005).

[20] J. B. Hartle and S. W. Hawking,Phys. Rev. D 28, 2960 (1983).

[21] N. J. Cornish, D. N. Spergel and G. D. Starkman, Phys. Rev. Letts. 77, 215 (1996) ; Class. Quantum Grav. 15, 2657 (1998).

[22] N. J. Cornish and D. N. Spergel, 'A small Universe after all ? ' - astro-ph/9906401.

[23] S. W. Hawking and N. G. Turok, Phys. Lett. B 425, 25 (1998), hep-th/9802030; N. G. Turok and S. W. Hawking, Phys. Lett. B 432, 271 (1998), hep-th/9803156. 